\title{
The Prediction of Metro Shield Construction Cost Based on a Backpropagation Neural Network Improved by Quantum Particle Swarm Optimization
}

\author{
Lanjun Liu $\mathbb{D}^{1},{ }^{1}$ Denghui Liu $\mathbb{D},{ }^{2}$ Han $W u\left(\mathbb{D},{ }^{3}\right.$ and Xinyu Wang $\mathbb{D}^{4}$ \\ ${ }^{1}$ School of Civil Engineering and Architecture, Wuhan Institute of Technology, Wuhan 430070, China \\ ${ }^{2}$ China Construction First Group Corporation Limited, Beijing 100161, China \\ ${ }^{3}$ School of Civil Engineering and Architecture, Wuhan University of Technology, Wuhan 430070, China \\ ${ }^{4}$ School of Architectural Engineering, Xinyang Vocational and Technical College, Xinyang 464000, China
}

Correspondence should be addressed to Denghui Liu; 293203@whut.edu.cn

Received 3 October 2020; Revised 23 November 2020; Accepted 10 December 2020; Published 19 December 2020

Academic Editor: Hugo Rodrigues

Copyright (c) 2020 Lanjun Liu et al. This is an open access article distributed under the Creative Commons Attribution License, which permits unrestricted use, distribution, and reproduction in any medium, provided the original work is properly cited.

\begin{abstract}
The prediction of construction cost of metro shield engineering is of great significance to project management. In this study, we used the rough set theory, a backpropagation (BP) neural network, and quantum particle swarm optimization (QPSO) to establish a prediction model for predicting the metro shield construction costs. The model accounts for the complexity of metro shield construction and the nonlinear relationship between the construction cost factors. First, the factors affecting the construction cost were determined by referring to the Chinese National Standards and analysing the engineering practice of typical metro shield projects. The rough set theory was used to simplify the system of influencing factors to extract the dominant influencing factors and reduce the number of input variables in the BP neural network. Since the BP neural network easily falls into a local minimum and has a slow convergence speed, QPSO was used to optimize the weights and thresholds of the BP neural network. This method combined the strong nonlinear analysis capabilities of the BP and the global search capabilities of the QPSO. Finally, we selected 50 projects in China for a case analysis. The results showed the dominant factors affecting the construction cost of these projects included ten indicators, such as the type of tunnelling machine and the geological characteristics. The determination coefficient, mean absolute percentage error, root mean square error, and mean absolute error, which are frequently used error analysis tools, were used to analyse the calculation errors of different models (the proposed model, a multiple regression method, a traditional BP model, a BP model optimized by the genetic algorithm, and the BP model optimized by the particle swarm optimization). The results showed that the proposed method had the highest prediction accuracy and stability, demonstrating the effectiveness and excellent performance of this proposed method.
\end{abstract}

\section{Introduction}

With the characteristics of energy-saving, land-saving, large transportation volume, low pollution, and low-operational risk, the metro plays an increasingly important role in the urban public transportation system [1]. Nowadays, developing countries, represented by China, are at the peak of urban rail transit construction. According to the Thirteenth Five-Year Plan issued by the Chinese government, by 2020 , the total length of the Chinese metro will reach $8,600 \mathrm{~km}$, and the investment in metro construction will reach 300 billion dollars. The construction of metro projects includes the tunnel and metro stations. The cost of tunnel construction often accounts for more than $40 \%$ of the total construction costs [2]. Shield construction technology has the advantages of a small impact on the surrounding environment, fast construction speed, excellent engineering quality, construction safety, environmental protection, and wide application range. Shield construction is the most commonly used method of urban metro tunnel construction [3]. Improving the accuracy and reliability of the cost 
prediction of shield construction ensures the smooth execution of metro construction projects and protects the economic interests of the owners, construction companies, and the public.

There are substantial differences in the definition of construction costs between different countries due to national regulations and different construction contracts. In this study, we selected the most authoritative definition of the contract unit price, which has been recommended by the International Federation of Consulting Engineers (FIDIC) and is accepted by the World Bank, to define the construction cost of the metro shield. The selection of the contract unit price also facilitates follow-up research. The total cost of metro shield construction comprises all costs paid by the owner during the metro shield construction, including direct costs, indirect costs, headquarters management fees, profits, taxes, tentative deposits, changes approved by engineers during the implementation process, and the amount claimed by the contractor [4]. The final cost of metro shield projects depends on the length of the project, but the unit price of the construction cost per kilometre $(\mathrm{km})$ is comparable. Therefore, the construction cost analysed in this article is the unit price of metro shield construction per $\mathrm{km}$.

According to the above definition, the components of the metro shield construction cost are relatively complex, and the influencing factors include the construction details, construction environment, project management, capital structure, and many other factors. Combined with engineering practice, it is evident that the influence of these factors on the construction cost of the metro shield is relatively complicated. On the one hand, the construction process has high degrees of mechanization and standardization; thus, the construction costs are related linearly to the project size [5]. On the other hand, many nonlinear and qualitative factors, such as hydrological conditions, geological conditions, environmental characteristics, and project management capabilities, have a nonlinear influence on the metro construction costs [6].

Many scholars have conducted in-depth research on construction cost prediction. Wang et al. [7] used an activity-based costing method to predict the supply chain cost of prefabricated building construction in an uncertain environment. Although the model could be used to determine the key factors to reduce the cost of the entire supply chain, the subjectivity and arbitrariness of the choice of the cost drivers led to poor cost forecasting performance. Chen [8] used multiple regression analysis to predict the cost of four construction projects. However, it was assumed that the relationship between the independent variable and the dependent variable was linear, affecting the accuracy of the prediction results. Tabei et al. [9] believed that the main factor leading to the large prediction error was the uncertainty of the factors, and a new method was proposed to predict the project cash flow in a fuzzy environment. However, this method requires a large amount of comprehensive statistical data to obtain consistent research results, adversely affecting the applicability of this method. Cheng et al. [10] used the golden section method, and the grey forecast method improved by dichotomy to establish a forecast model. The method provided good prediction accuracy for the case study, but the grey forecast method requires uniformity of the original data. In addition, the above methods either rely significantly on professional knowledge or are only suitable for specific situations; thus, they do not have modelling and analysis flexibility.

The emergence of artificial intelligence algorithms, such as artificial neural networks (ANNs), provides a new approach for solving the complex problems of predicting the metro shield construction cost. ANNs can approach any nonlinear continuous function with arbitrary precision and are well suited for solving the highly nonlinear problem of cost prediction [11]. ANNs have strong self-learning and self-adaptive abilities. They can extract information from input and output data and store it in the weights of the network; thus, they possess good generalization ability [12]. In addition, ANNs are able to use a limited amount of data obtained in the field, rather than using large amounts of statistical data so that a data-driven method can be used to predict the construction costs.

A backpropagation (BP) neural network is a multilayer feedforward network trained by an error BP algorithm, and it is the most widely used type of ANN [11]. However, traditional BP neural networks may have the following shortcomings when applied to the analysis of complex systems. (1) The traditional BP neural network algorithm is a local search optimization method, which easily falls into local extremes, and the weights converge to the local extremes, a small sample size resulting in network training failure [13]. (2) The BP neural network is very sensitive to the initial network weights. Initializing the network with different weights tends to converge to different local minima [14]. Therefore, a high-precision model has to be developed to improve the traditional BP neural network.

Some scholars have used a genetic algorithm (GA) and particle swarm optimization (PSO) to improve the traditional BP model. The initial weights and thresholds of the BP neural network are optimized by these metaheuristic algorithms to achieve higher accuracy than other methods. Wen et al. [15] established a construction cost prediction model for a power transmission and transformation project using a BP model optimized by a GA. However, the GA algorithm had some problems, such as depending on the initial population selection, a slow convergence speed, and many parameter settings, resulting in relatively low accuracy of the model. Liu and Chi [16] used the PSO to optimize the weights and thresholds and establish a cost estimation model for a government investment project. Compared with classical metaheuristic algorithms, such as a GA, the PSO algorithm has the advantages of simple operation, fast convergence speed, and few parameter settings [17]. However, PSO has a limited search space and easily falls into a local extremum $[18,19]$. The reason is that, in the classical PSO, the particles have a tendency to orbit rather than converging to zero, and the particles have a maximum speed limit during the search; thus, the search area is limited in each search. Sun et al. [18] proposed the novel quantum particle swarm optimization algorithm (QPSO), which is 
based on the PSO algorithm. During particle convergence, the particle keeps approaching the point until it falls inside. From the perspective of quantum mechanics [19], an attractive potential exists at the midpoint of the convergence process, attracting the particles to converge so that the whole group is aggregated. Particles in the quantum bound state can appear at any space point with a certain probability, which ensures good convergence performance and global search ability of the QPSO. Xu et al. [20] used the QPSO for the optimization of synthetic and actual hyperspectral datasets. The experimental results showed that the QPSO method obtained better results than vertex component analysis (VCA) and the standard PSO in remote sensing. Sun et al. [21] solved the problem of designing an optimal twodimensional zero-phase infinite impulse response (IIR) digital filter using QPSO. Before analysing unmanned aerial vehicle (UAV) route planning, $\mathrm{Fu}$ et al. [22] tested various optimization algorithms, such as GA, differential evolution (DE), the standard PSO, the phase-coded PSO, the QPSO, and other optimization algorithms. The results showed that the computational power of QPSO was better than that of GA, DE, and classic PSO algorithms. Therefore, in this study, we used the QPSO to optimize the BP model.

Many factors affect the construction cost of metro shields. If all factors are included in the input of the BP model, the computational burden for training the neural network will be high, and the prediction accuracy will be reduced. In the selection of input variables for the BP model, most studies used threshold analysis with an arbitrary threshold to select the input variables for the BP model [23]. This selection method is affected by subjective factors, causing information redundancy or loss. The accuracy of neural network prediction can be increased by using a suitable method for selecting the important factors affecting the construction cost of metro shields as model inputs.

Rough set theory is a mathematical analysis tool to describe the completeness and uncertainty of data [24]. The method simplifies the data, obtains the minimum expression of knowledge while retaining key information, and identifies and evaluates the dependence between the data. Shiau et al. [25] successfully used rough set theory to screen factors affecting transportation sustainability in Taiwan. Cheng et al. [26] used the rough set theory to select and analyse 21 indicators affecting regional green competitiveness and formulate regional green competitiveness indicators. Ge et al. [27] extracted spatial relationship indicators using rough set theory to describe the complex relationship between geographical phenomena and the environment. Many factors affect the cost of subway shield construction. If we regard them as a knowledge expression system and use a fuzzy rough set method to analyse various factors that affect the cost of subway shield construction, we can reduce the number of variables and obtain many important attributes influencing the construction cost of a subway shield. These factors can be used as input to the neural network to optimize the input variables, and the importance of each attribute in the cost prediction of subway shield construction can be determined.
Against this background, we established a prediction model based on a BP neural network improved by the QPSO. This paper provided the following contributions. (1) This paper determined the factors affecting the construction cost and provided details on the data acquisition or calculation method for each factor. We used the rough set theory to select the main influencing factors affecting the construction cost of 50 metro shield projects in a case analysis. This approach not only handled scientifically the complexity of metro shield construction and the numerous factors affecting construction costs but also substantially reduced the input variables of the BP neural network prediction model. (2) This paper used the BP algorithm optimized by the QPSO to establish a cost prediction model for metro shield construction. This method combined the global convergence ability of the QPSO and the speed of the local search of the BP algorithm, thus improving the stability of the training process of the BP network and the accuracy of the training results. (3) The error analysis of the case study showed that the BP neural network optimized by QPSO had higher prediction accuracy and stability than the multiple regression method, the conventional BP model, the BP model optimized by GA, and the BP model optimized by PSO.

The remaining sections of this article are organized as follows. Section 2 constructs a detailed construction cost impact index system of subway shield engineering and the cost prediction model based on the BP optimized by the QPSO. Section 3 is the case analysis, which analyses the construction costs of 50 subway shield projects in detail. Section 4 is the discussion. Section 4 details the error and stability analysis of different algorithms and also analyses the influence of the topological structure of the BP neural network. Section 5 is the conclusion. After a brief analysis of the limitations of the research results in this paper, Section 5 summarizes the research results of this article and points out further research directions.

\section{Materials and Methods}

\subsection{The Indicator System of the Construction Cost of Metro Shield Engineering}

2.1.1. Analysis of the Influencing Factors of Metro Shield Construction Cost. We use a project breakdown structure (PBS) to decompose the construction process and to analyse accurately and comprehensively the factors affecting the construction cost of subway shield engineering [28]. Subsequently, we selected the factors affecting the construction cost of metro shield engineering. By referring to the Urban Rail Transit Project Investment Estimation Indicators (GCG 101-2008) promulgated by the Ministry of Housing and Urban-Rural Development of the People's Republic of China, and the Regulations on Capital Construction Project Construction Cost Management promulgated by the Ministry of Finance of the People's Republic of China (Caijian (2016) No. 504), we decomposed the construction aspects into four categories: project aspects, construction conditions, technical standards, and project management. 
(1) Project Aspects. Metro shield engineering refers to the installation of the shield and does not include other aspects of construction, such as connecting the passages and pump houses, reinforcement of risk sources, construction monitoring, and evacuation platforms. The metro shield project includes three parts: the selection of the shield machine, the shield construction at the start or exit stage, and the construction in the shield section. With reference to the engineering practice of the Chengdu Metro Line 11, the Wuhan Metro Line 8, the Tianjin Metro Line 7, and the Wuhan Metro Line 21, we analysed the engineering aspects of the metro shield project. The selection of the shield machine has not only a significant impact on the smooth completion of construction but also on the cost. A shield machine adapted to the engineering and geological characteristics can complete the task quickly with high quality and low risk. Thus, the shield machine, which costs more than 10 million dollars, substantially reduces the amortization cost of the project. At present, there are two types of shield machines: an Earth pressure balance shield machine and a mud-water balance shield machine, which are adapted to different geological characteristics. The permeability coefficient of the stratum is not only an important geological factor but also a vital parameter to determine the selection of the shield machine [29]. According to the national standard of the People's Republic of China (Code for Construction and Acceptance of Shield Tunnelling Methods (GB 50446-2017)), when the ground seepage coefficient exceeds $10^{-4} \mathrm{~m} / \mathrm{s}$, the mud-water balance shield machine should be considered. When the seepage coefficient is less than $10^{-7} \mathrm{~m} / \mathrm{s}$, the Earth pressure balance shield machine is considered. When the ground seepage coefficient is between the two values, either model can be used, depending on the conditions. The minimum radius of the tunnel line, the maximum slope, and the clearance in the tunnel also affect the selection of the shield machine. The minimum radius of the line should match the size of the shield machine, and an appropriate shield size should be selected according to the clearance in the tunnel. It is worth mentioning that the geological characteristics affect the selection and the efficiency of the shield machine. Therefore, they also have a significant impact on the shield construction costs.

In the initial stage of shield construction, the launch shaft is first established and reinforced, followed by the assembly, debugging, and trial excavation. After trial tunnelling has been performed successfully, normal tunnelling begins. During the trial tunnelling process, it is necessary to evaluate the tunnelling parameters of the shield machine to find the optimal parameters. Therefore, the construction cost of the trial tunnelling stage is often significantly higher than that of the formal construction stage. The construction cost of the trial tunnelling stage is proportional to the length of the trial tunnel. The cost of the shield machine's entry and exit work is primarily determined by the number of times the machine enters and exits the field; the cost is generally calculated based on the number of times.

In the construction of the shield section, in addition to hydrogeological conditions affecting the construction cost, segment engineering is the main factor. Different segment thicknesses entail different concrete requirements, and the concrete costs account for a large proportion of the total material cost. It is worth mentioning that since the construction cost analysed in this study is based on the unit price per $\mathrm{km}$ for metro shield construction, the length of the shield section has a negligible effect on the unit price.

Based on the above analysis, the basic engineering features of the metro shield included the type of shield machine (X1), the permeability coefficient of the stratum (X2), the minimum radius of the tunnel line (X3), the maximum slope (X4), the clearance in the tunnel (X5), the geological characteristics (X6), hydrological conditions (X7), predrilling length (X8), the number of shield machine entrances and exits (X9), and the segment thickness (X10).

(2) Construction Conditions. Similar to the construction cost, the construction period is also affected by many factors. Generally, a reduction in the construction period increases the direct costs of construction, whereas the indirect costs are reduced. In contrast, an increase in the construction period may lead to a reduction in direct costs but an increase in indirect costs. Therefore, the construction period is selected as an influencing factor. Due to differences in the area of metro projects, the area directly affects the wages and the price of materials and machinery required by the project. Since different regions have different fee collection standards, the location of the project affects the construction cost.

Metro projects are often built in cities or suburbs, where there are already many buildings and municipal pipelines. Certain measures are required during shield construction to protect these buildings and municipal pipelines. Therefore, the more complicated the surrounding environment of a subway project, the more engineering measures are required and the higher the construction cost is. The seismic fortification intensity (seismic capacity of metro projects) is also an important factor affecting construction costs. Different project standards are used for different seismic requirements. The higher the seismic fortification intensity, the higher the engineering standards and quality standards are. Therefore, we regard the seismic fortification intensity as a basic engineering feature.

We select the construction period (X11), environmental characteristics (X12), seismic fortification intensity (X13), and other related factors.

(3) Technical Standards. According to the Code for the Design of Metro Systems (GB 50157-2013), which is the national standard of the People's Republic of China, the current design speed of metro systems is divided into several grades, such as $60,80,100,120$, and 160 . However, most of the lines are designed for a speed of 80 due to the station distance, train performance, and overall cost. If the designed speed is high, but the station distance is small, the train has to slow down to enter the station before. This approach does not represent an advanced design of the system and is a waste of resources. If the train runs fast, the higher the requirements for the train performance and the curve radius of the line, the higher the construction cost is. However, the accessibility is 
low, and the travel needs of residents in some areas are not considered, which is unreasonable. Therefore, the design speed (X14) is selected as a basic engineering feature that affects the construction cost of the metro shield project.

At present, common structural forms of tunnel construction include double-line double tunnels and doubleline single tunnels. In theory, double-line single tunnels are more economical because only one excavation is needed, and the cost is reduced [30]. However, the situation is the opposite in practical applications. Due to the need for high technology and a sound geological structure for large crosssection excavation, the construction of a double-line single tunnel is often more expensive. Therefore, a single-line single tunnel is typically used in many more cases. We select the tunnel structure (X15) as a basic engineering feature that affects the construction cost of metro shield engineering.

(4) Project Management. Project management includes the management ability of the owner (X16), the management ability of the construction company (X17), and the financing structure (X18). The stronger the project management capabilities of the owners and construction companies are during the life cycle of the metro tunnel project, the better the allocation and coordination of resources are. Scientific decisions are made to facilitate project execution and lower construction costs. Due to extensive investments in metro projects, it requires a long time to recover the costs to benefit the public. When metros are constructed in developing countries, method such as Build-Transfer (BT), BuildOperate-Transfer (BOT), Public-Private Partnership (PPP), Build-Own-Operate (BOO), and other financing models are used [31]. These approaches save government interest expenses, thereby indirectly reducing construction costs. Different financing models have different cost inputs; therefore, the financing models affect project management.

2.1.2. Data Collection. According to the analysis in Section 2.1.1, this section presents the data collection methods of the 19 primary and secondary indicators. X6, X7, X16, and X17 are qualitative indicators, whose scores are obtained from a questionnaire survey of experts. The other indicators are quantitative indicators, and the data are obtained by referring to design documents or field surveys. We focus on the practices used during the construction of Chengdu Metro Line 11, Wuhan Metro Line 8, Tianjin Metro Line 7, and Wuhan Metro Line 21. This section provides a detailed description of the indicators and how to obtain the data, which is convenient for subsequent research on the selection of the index system and case analysis based on rough set theory.

Shield Machine Type (X1). The soil pressure balance shield machine has a score of 1 , the mud-water balance shield machine has a score of 2 , and the other types of shield machines have a score of 3 . This indicator has no unit.

The Permeability Coefficient of the Formation (X2). The score of this indicator can be obtained by consulting the geological survey or design documents, and the unit is $\mathrm{m} / \mathrm{s}$. The score can also be obtained from an on-site survey; the calculation formula [32] is

$$
\kappa=\frac{k \rho g}{\eta}
$$

where $\kappa$ is the permeability coefficient of the formation, $k$ is the permeability of the porous medium, $\eta$ is the dynamic viscosity coefficient, $\rho$ is the fluid density, and $g$ is the acceleration of gravity. A field test is used to obtain the values of $k, \rho$, and $\eta$ so that the permeability coefficient of the formation can be calculated.

The Minimum Radius of the Tunnel Line (X3). The score of this indicator can be obtained by consulting the design documents or conducting an on-site investigation; the unit is $m$.

Maximum Slope (X4). The score of this indicator can be obtained by consulting the design documents or conducting an on-site investigation. The unit of this indicator is \%o.

Clearance in the Tunnel (X5). The score of this indicator can be obtained by consulting the design documents or conducting an on-site investigation; the unit is $m$.

Geological Features (X6). According to the Technical Code for the Investigation of Geotechnical Engineering (YS 5202-2004), which is the industry standard of the People's Republic of China, the geological factors that impact subway shield engineering and typical underground engineering construction include the topography, stratum lithology, geological structure, earthquake probability, hydrogeology, natural building materials, and undesirable physical geological phenomena, such as karst, high probability of landslide, collapse, sand liquefaction, and foundation deformation. This qualitative indicator is complex and comprehensive. We divide the impact of the geological features on the construction cost into five levels: (1) very unfavourable, (2) unfavourable, (3) general, (4) favourable, and (5) very favourable.

Hydrological Conditions (X7). Hydrogeological conditions refer to the impact of various changes and movements of groundwater within the scope of the subway shield project on the construction cost of the shield project. We divide the impact of hydrological conditions on the construction cost into five levels: (1) very unfavourable, (2) unfavourable, (3) general, (4) favorable, and (5) very favourable.

Predrilling Length (X8). The score of this indicator can be obtained by consulting design documents or project management documents, and the unit is $m$.

The Number of Shield Machines Entering and Exiting the Field (X9). The score of this indicator can be obtained by consulting the design documents or conducting an on-site investigation. There is no unit for this indicator. 
Segment Thickness (X10). The score of this indicator can be obtained by consulting design documents or project management documents, and the unit is $m$.

Construction Period (X11). The score of this indicator can be obtained by consulting the design documents or conducting an on-site investigation; the unit is day (d).

Environmental Characteristics (X12). We divide the impact of the subway shield project on the construction cost into five levels: (1) very unfavourable, (2) unfavourable, (3) general, (4) favourable, and (5) very favourable.

Seismic Fortification Intensity (X13). According to the National Standard of the People's Republic of China Standard for the Classification of Seismic Protection of Building Construction (GB 50223-2008), the design basic seismic acceleration value is selected as the score of this index. The unit is gravitational acceleration $(g)$.

Design Speed (X14). The score of this indicator can be obtained by consulting the design documents, and the unit is $\mathrm{km} / \mathrm{h}$.

Tunnel Structure (X15). At present, the common structural forms of tunnel construction are mainly double-line double tunnels and double-line single tunnels. The scores are 1 and 2, respectively.

The Project Management Ability of the Owner (X16). Generally, the higher the management ability of the owner, the better the cost control is. The management ability of the contractor is determined according to the quality of the construction unit. This is a qualitative indicator. Since the indicator is dimensionless, the levels are (1) excellent, (2) good, (3) moderate, and (4) poor.

The Project Management Ability of the Construction Companies (X17). Generally, the higher the management ability of the contractor, the better the cost control is. The management ability of the contractor is determined according to the quality of the construction unit. This is a qualitative indicator. Since the indicator is dimensionless, the levels are (1) excellent, (2) good, (3) moderate, and (4) poor.

The Financing Structure of the Project (X18). There are currently four common financing structures for subway projects. The score of BT is 1 , the score of BOT is 2 , the score of PPP is 3, the score of $\mathrm{BOO}$ is 4 , and that of the other modes is 5 .

2.1.3. Index Screening Based on the Rough Set Theory. Rough set theory is a mathematical theory proposed by the Polish mathematician Pawlak that can deal with uncertain, incomplete, and incompatible information and knowledge [24]. Attribute reduction based on the rough set is accomplished by deleting unimportant or irrelevant and redundant attributes while maintaining the classification or decision-making ability of the information system and deriving the classification or decision-making of the problem. An information system can be represented by a 4tuple:

$$
S=(U, A, V, f)
$$

where $U$ is the universe of discourse, $A=C \cup D=\left(a_{1}, a_{2}, \ldots, a_{n}\right)$ is a limited set of nonempty attributes, $C \cap D=\varnothing, C$ are conditional attribute sets, $D$ is a decision attribute set, and $A$ is a set of attribute values. Function $A$ is used to assign a corresponding attribute value to each object in the universe of discourse. The knowledge expression system with conditional attributes and decision attributes is collectively called a decision table, in which the rows represent the records of the object and the columns represent the attributes of the object [25].

Definition 1. The degree of contribution of the conditional attributes $C$ to the decision attributes $D$ is described as

$$
\gamma_{C}(D)=\frac{\operatorname{POS}_{C}(D)}{|U|}
$$

where $\operatorname{POS}_{C}(D)$ is called the positive domain of $D$ with respect to $C$, which describes the set of all elements in $U$ that can be accurately classified into Class $A$ based on knowledge of $U \mid D$. The $\gamma_{C}(D)$ represents the proportion of objects that can be classified into Decision Class $C$ under conditional attribute $U \mid D$. This defines the degree of contribution of the conditional attributes to the decision attributes.

Definition 2. Required attributes and unnecessary attributes. Suppose there is $q \in C, C \subseteq A$ in the information system $S$. If $\operatorname{POS}_{C}(D) \neq \operatorname{POS}_{(C-q)}(D)$, it is said that $q$ is a necessary attribute of $C$ and must be retained. Otherwise, $q$ is an unnecessary attribute of $C$ and can be deleted. If both $q \in C$ and $C$ are necessary attributes of $C$, it can independently form a set of features expressing the classification of information systems. In this case, $C$ is called independent; otherwise, it is dependent.

\section{Definition 3. Reduction and core.}

In the information system $S, E \subseteq C \subseteq A$ and $E$ are independent and satisfy $\left(\gamma_{E}(D)=\gamma_{C}(D)\right) \wedge(\forall E \subset E) \Rightarrow($ $\left.\gamma_{E^{\prime}}(D) \neq \gamma_{C}(D)\right)$. $E$ is called a reduction of $C$, which is denoted as $\operatorname{Red}(C)$. The set of necessary attributes of $C$ forms the core of $C$, which is denoted as $\operatorname{Core}(C)=\cap \operatorname{Red}(C)$. An information system usually contains multiple reductions. If $E$ is independent, then $E$ is the smallest set that maintains the classification ability of the universe $U[25,26]$.

In this study, the Rosetta software is selected to screen the indicators of the metro shield construction cost. The software is a logical analysis and data processing tool based on rough set theory [33]. It was developed in cooperation with the Department of Computer and Information Science at the Norwegian University of Science and Technology and the Institute of Mathematics at the University of Warsaw, Poland. The software integrates a variety of data preprocessing methods, such as data complementation, discretization, and other functions. It can also perform operations such as rule acquisition and 
index reduction, as well as data mining and knowledge acquisition [34].

The Chengdu Metro Line 11 (16 shield sections), Wuhan Metro Line 8 (9 shield sections), Tianjin Metro Line 7 (13 shield sections), Wuhan Metro Line 21 (12 shield sections), and others were used in the case study. Quantitative index data were obtained by consulting design materials and field surveys and using other methods. In addition, 100 experts were invited to score each qualitative index. The criteria for selecting experts are work unit, working years, professional titles, and educational background. After two rounds of questionnaires, a total of 72 valid questionnaires were obtained. For simplification, 50 questionnaires were randomly selected. Cronbach's alpha reliability coefficient of the questionnaires was greater than 0.7 , so the questionnaire system was credible [34]. In fact, this paper randomly selected three times, and the values of Cronbach's alpha of the three questionnaires were $0.7831,0.831$, and 0.847 , which were all greater than 0.7 . All index score data are shown in Table 1. Due to the length of the paper, Table 1 only lists some data.

The objective was to maintain the classification ability of the knowledge system. Rosetta's built-in attribute reduction algorithm was used to delete the irrelevant or unimportant secondary indicators and to extract the sensitive predictive indicators of the metro shield construction costs. We extracted the type of shield machine (X1), the geological characteristics (X6), the number of entrances and exits of the shield machine (X9), and the segment thickness (X10) as the attributes of the project aspect. The construction period (X11) and environmental characteristics (X12) were used as the attributes of the construction conditions. The design speed (X14) and tunnel structure (X15) were used as the attributes of the technical standards. The project management ability of the owner (X16), the project management ability of the construction company (X17), and the financing structure of the project (X18) were used as the attributes of project management. In addition, since the design speed in the current metro engineering practice is $80 \mathrm{~km} / \mathrm{h}$, we deleted the design speed (X14). We discuss the reason for deleting this indicator in Section 3.3.

2.1.4. Development of the Indicator System. The primary and secondary indicators affecting the construction cost of metro tunnel engineering are shown in Table 2.

\subsection{The Construction Cost Prediction Model Based on the} QPSO-BP Algorithm. The QPSO algorithm has the advantages of speed and a strong global search, unlike the BP model. In addition, QPSO does not require specific characteristic information of the problem, such as derivative gradient information. Therefore, the QPSO and BP models can be combined to take advantage of the generalization ability of the BP model and the ability of the QPSU to optimize the initial weights and thresholds of the layers of the network. This integration improves the neural network's convergence speed and learning ability.
2.2.1. The BP Model. The BP network was proposed in 1986 by a team of scientists headed by Rumelhart and McClelland. The BP neural network has self-learning and adaptive capabilities. When the output value is different from the expected value, the error is calculated. In this case, the neuron runs the information transmission process opposite to the learning process [35]. Since the principle of the BP neural network learning algorithm is the gradient descent method, the output result, in which the error meets the requirements, can be obtained by repeated training. At present, the threelayer BP network is the most commonly used network. The model structure is shown in Figure 1 , where $X_{1}, X_{2}, \cdots, X_{n}$ are the input values and $Y_{1}, Y_{2}, \cdots, Y_{m}$ are the output results.

2.2.2. The QPSO Model. PSO is an optimization method that emerged in the 1990s. It has the advantages of simple structure, easy programming, and small amount of calculation. The search space of the standard PSO is limited, and it is easy to fall into the local extremum [36]. In the standard PSO, the convergence of particles is realized in the form of orbit, and the maximum speed of particles is limited in the process of searching. So, the standard PSO cannot globally converge with probability [37]. In the process of particle convergence in QPSO, particle $i$ keeps approaching $P_{i}$ until it falls within $P_{i}$. There is an attractive potential at $P_{i}$ during the convergence process, which attracts particles to converge to $P_{i}$ and makes the whole population have aggregation [18]. Particles in the quantum bound state can appear at any space point with a certain probability [20]. Therefore, particles can search in the whole solution space and thus have better convergence. This is the theoretical reason why QPSO has better computational performance than PSO.

Compared with the standard PSO, the QPSO has a different evolutionary search strategy and eliminates the unnecessary velocity vector in the evolutionary equation. In addition, the QPSO has a simpler evolutionary equation and fewer parameters and is easier to control; thus, QPSO has better global search performance.

In a population with $M$ particles, each particle moves in an $D$-dimensional space. The velocity and position vectors of the $i$ th particle are $X_{i, n}=\left(X_{i, n}^{1}, X_{i, n}^{2}, \ldots, X_{i, n}^{D}\right)$ and $V_{i, n}=\left(V_{i, n}^{1}, V_{i, n}^{2}, \ldots, V_{i, n}^{D}\right)$. In the classical PSO, the trajectory of particles [17] is updated using

$$
\begin{aligned}
& V_{i, n+1}^{j}=V_{i, n}^{j}+c_{1} r_{i, n}^{j}\left(P_{i, n}^{j}-X_{i, n}^{j}\right)+c_{2} R_{i, n}^{j}\left(G_{i, n}^{j}-X_{i, n}^{j}\right), \\
& X_{i, n+1}^{j}=X_{i, n}^{j}+V_{i, n+1}^{j},
\end{aligned}
$$

where $i=1,2, \ldots, M, j=1,2, \ldots, D, c_{1}$ and $c_{2}$ are acceleration factors, $r_{i, n}^{j}$ and $R_{i, n}^{j}$ are random numbers in the range of $(0,1), V_{i, n}^{j} \in\left[-V_{\max }, V_{\max }\right], P_{i, n}=\left(P_{i, n}^{1}, P_{i, n}^{2}, \ldots, P_{i, n}^{D}\right)$ is the individual optimal position of particle $i$, and $G_{n}=\left(G_{n}^{1}, G_{n}^{2}, \ldots, G_{n}^{D}\right)$ is the global optimal position.

In the QPSO algorithm, the position and velocity vectors of each particle are replaced by wave functions [19]. The trajectory of the particles is updated using equations (6)-(8) [19-22]: 
TABLE 1: Scores of the indices and the actual construction cost.

\begin{tabular}{|c|c|c|c|c|c|c|c|c|c|c|}
\hline No. & Actual cost & $\mathrm{X} 1$ & $\mathrm{X} 2$ & $\mathrm{X} 3$ & $\mathrm{X} 4$ & $\ldots$ & $\mathrm{X} 15$ & $\mathrm{X} 16$ & $\mathrm{X} 17$ & $\mathrm{X} 18$ \\
\hline Unit & Millions/km & - & $\mathrm{m} / \mathrm{s}$ & $\mathrm{m}$ & $\%$ & $\ldots$ & - & - & - & - \\
\hline 1 & 281.62 & 1 & $7.0 \times 10^{-8}$ & 250 & 6 & $\ldots$ & 1 & 1 & 2 & 3 \\
\hline 2 & 473.78 & 1 & $9.2 \times 10^{-5}$ & 280 & 4 & $\ldots$ & 1 & 3 & 1 & 3 \\
\hline 3 & 867.03 & 2 & $4.3 \times 10^{-4}$ & 300 & 4 & $\ldots$ & 1 & 2 & 1 & 2 \\
\hline 4 & 387.49 & 1 & $1.0 \times 10^{-5}$ & 250 & 6 & $\ldots$ & 1 & 2 & 2 & 2 \\
\hline$\vdots$ & $\vdots$ & $\vdots$ & $\vdots$ & $\vdots$ & $\vdots$ & $\vdots$ & $\vdots$ & $\vdots$ & $\vdots$ & $\vdots$ \\
\hline 46 & 383.69 & 1 & $3.7 \times 10^{-5}$ & 250 & 4 & $\ldots$ & 2 & 2 & 1 & 3 \\
\hline 47 & 738.50 & 1 & $4.0 \times 10^{-5}$ & 250 & 4 & $\ldots$ & 2 & 3 & 1 & 3 \\
\hline 48 & 424.31 & 1 & $2.8 \times 10^{-7}$ & 300 & 6 & $\ldots$ & 2 & 2 & 1 & 3 \\
\hline 49 & 337.47 & 1 & $6.4 \times 10^{-8}$ & 250 & 6 & $\ldots$ & 1 & 2 & 1 & 3 \\
\hline 50 & 549.34 & 1 & $8.5 \times 10^{-6}$ & 250 & 6 & $\ldots$ & 1 & 1 & 1 & 3 \\
\hline
\end{tabular}

TABLE 2: The indicators affecting the metro tunnel construction cost.

\begin{tabular}{|c|c|c|}
\hline Primary indicators & Secondary indicators & Type of Indicator \\
\hline Project aspects & $\begin{array}{c}\text { Type of shield machine (X1) } \\
\text { Geological features (X6) } \\
\text { Number of times of shield machine entering and leaving the site (X9) } \\
\text { Segment thickness (X10) }\end{array}$ & $\begin{array}{l}\text { Quantitative } \\
\text { Qualitative } \\
\text { Quantitative } \\
\text { Quantitative }\end{array}$ \\
\hline Construction conditions & $\begin{array}{c}\text { Construction period (X11) } \\
\text { Environmental characteristics (X12) }\end{array}$ & $\begin{array}{l}\text { Quantitative } \\
\text { Qualitative }\end{array}$ \\
\hline Technical standard & Tunnel structure (X15) & Quantitative \\
\hline Project management & $\begin{array}{c}\text { Project management ability of the owner (X16) } \\
\text { Project management ability of the construction company (X17) } \\
\text { Financing structure of the project (X18) }\end{array}$ & $\begin{array}{l}\text { Qualitative } \\
\text { Qualitative } \\
\text { Quantitative }\end{array}$ \\
\hline
\end{tabular}

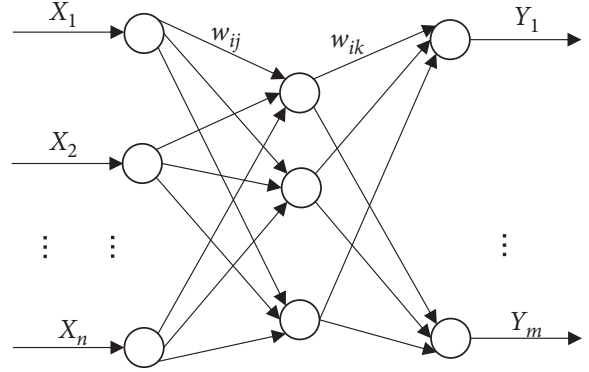

Figure 1: The structure diagram of the BP neural network.

$$
\begin{aligned}
X_{i, n+1}^{j} & =P_{i, n}^{j} \pm \alpha \cdot\left|X_{i, n}^{j}-C_{i, n}^{j}\right| \cdot \leq \operatorname{In}\left(\frac{1}{u_{i, n+1}^{j}}\right), \\
C_{n}^{j} & =\left(\frac{1}{M}\right) \sum_{i=1}^{M} p_{i, n}^{j}, \\
P_{i, n}^{j} & =\varphi_{i, n}^{j} \cdot P_{i, n}^{j}+\left(1-\varphi_{i, n}^{j}\right) \cdot G_{n}^{j},
\end{aligned}
$$

where $C_{n}=\left(C_{n}^{1}, C_{n}^{2}, \ldots, C_{n}^{D}\right)$ is the average optimal position, which is determined by averaging the individual optimal positions of all particles, $\varphi_{i, n}^{j}$ and $u_{i, n+1}^{j}$ are random numbers uniformly distributed in $(0,1)$, and $\alpha$ is the scaling coefficient that controls the convergence speed. It is the only control parameter in the QPSO algorithm, except for the population size and iteration times.
2.2.3. The Prediction Model Based on the QPSO-BP. The flowchart of the prediction model based on the QPSO-BP is shown in Figure 2.

The main steps of using QPSO to optimize the weights and thresholds of the BP neural network are as follows:

Step 1. Data collection and normalization.

The numerical dimensions differ for the influencing factors, causing a decrease in the convergence speed when using the BP and QPSO algorithms and affecting the accuracy of the model [38]. Equation (9) was used [38] to normalize the data to obtain a range from 0 to 1 :

$$
x_{i}^{*}=\frac{x_{i}-x_{\min }}{x_{\max }-x_{\min }}-1
$$

where $x_{i}$ is the original data, $x_{\max }$ is the maximum value in the original data sequence, $x_{\min }$ is the minimum value in the original data sequence, and $x_{i}^{*}$ is the normalized data.

Step 2. Determining the input and output sample sets of the neural network and the topology of the neural network.

In the QPSO algorithm, the first-generation population is initialized within the range of $[0,1]$, and the size of the initial particle swarm population $M$, the scaling factor $\alpha$, and the maximum number of iterations $N$ are set.

Step 3. Determining the coding scheme of the neural network weights and thresholds. 


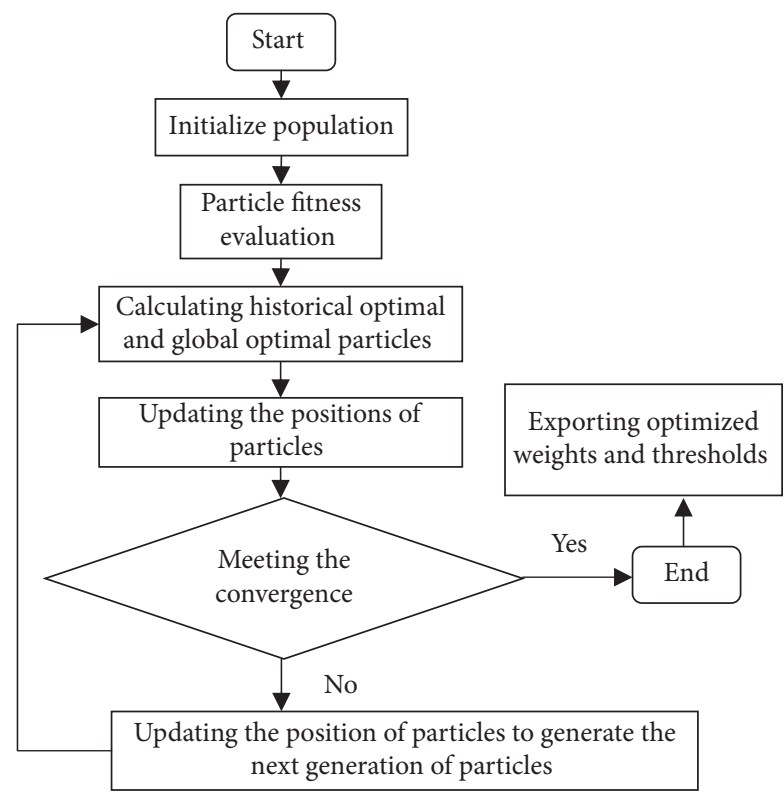

FIGURE 2: The flowchart of the prediction model based on the QPSO-BP.

We use a threshold or a weight to correspond to one dimension in the particle vector. For a specific neural network, if the input has $n$ dimensions, the number of hidden layers is $l$, the number of nodes in each corresponding hidden layer is $k_{i}$, and the output dimension is $m$; then, the dimension of the particles can be obtained using the following equation $[13,15]$ :

$$
D=n k_{1}+\sum_{i=1}^{l-1} k_{i} k_{i+1}+k_{l-1} m+\sum_{i=1}^{l} k_{i}+m .
$$

Step 4. Recoding the population individuals of the quantum particle swarm into the weights and thresholds of the neural network.

Step 5. Selecting the appropriate fitness function according to the neural network principle and input and output sample set.

The objective is to minimize the difference between the expected output of the network and the actual output. The fitness function is expressed as[13]

$$
f(x)=\sum_{i=1}^{s}\left(y_{i}-\widehat{y}_{i}\right)^{2}
$$

where $s$ is the number of samples, $y_{i}$ is the actual output of sample $i$, and $\hat{y}_{i}$ is the predicted output of sample $i$.

Step 6. Updating the particles according to equations (6) to (8).

Step 7. Evaluation of the particles and calculation of the fitness value using equation (11).

Step 8. If the end conditions are met, the optimization ends and the weights and thresholds at this time are used as the initial weights and thresholds of the neural network. Otherwise, return to Step (4).

\section{Case Analysis}

3.1. Obtaining and Processing Forecast Data. We selected four metro projects of the China State Construction Group Corporation for the case analysis. They include the Chengdu Metro Line 11 with 16 shield tunnels, Wuhan Metro Line 8 with 9 shield tunnels, Tianjin Metro Line 7 with 13 shield tunnels, and Wuhan Metro Line 21 with 12 tunnels. Table 1 shows the 50 sets of sample data. The time factors and geographic factors have a significant impact on the cost of the building material, labor, and machinery; therefore, they also have a significant impact on the construction costs, and it is necessary to adjust the 50 sets of construction cost data based on geography and time. It is worth mentioning that, in Table 1, the actual construction costs represent the adjusted data.

(1) We use Chengdu City, Sichuan Province, China as a benchmark and use the regional adjustment coefficient for the adjustment. According to the Standard method of measurement for public utilities works (GB 50857-2013), it can be calculated by the following equation:

$$
A=\frac{\sum_{i=1}^{n} D_{r i} * P_{r i}}{\sum_{i=1}^{m} D_{0 i} * P_{0 i}}
$$

where $n$ is the total number of similar local projects, $m$ is the total number of similar projects in other regions, $D_{r i}$ is the unit price of similar local projects, $P_{r i}$ is the weight of these projects. $P_{r i}=G_{r i} / \sum_{i=1}^{n} G_{r i}$, $G_{r i}$ is the number of similar projects, and $D_{0 i}$ and $P_{0 i}$ are the unit price and weight of the same types of project in other regions.

(2) Based on the cost index of 2018, the time adjustment is carried out by using the cost index adjustment. According to the Standard method of measurement for public utilities works (GB 50857-2013), it can be calculated by the following equation:

$$
B=\frac{\sum_{i=1}^{n} D_{r i} * P_{r i}}{\sum_{i=1}^{m} D_{0 i} * P_{0 i}}
$$

where $m$ is the total number of similar projects in 2018, $n$ is the total number of similar projects in other years, and $D_{r i}$ is the unit price of similar projects in the reporting period.

3.2. Forecast Results. Two sets of data were required for the BP neural network to establish the construction cost prediction model of metro shield engineering, i.e., the training set and test set. The training set data was used to train the neural network, and the test set data was used to validate the neural network. In this study, the training set consisted of 55 randomly selected datasets, and the rest of the data 
represented the test set. The ratio of training data to test data was $90 \%$ to $10 \%$.

The parameter settings of the BP neural network $[15,16]$ were as follows: the number of training times was 1000 , the learning rate was 0.1 , and the training target was 0.001 . The calculation parameters of QPSO $[20,21]$ included the following: the number of iterations was 1000 , the population size was 100 , the scaling factor was 0.4 , and the maximum error of the iteration termination was 0.00001 .

The convergence curve of the fitness function of the QPSO is shown in Figure 3. The QPSO model converged after about 150 generations.

The results of the optimization calculation process of QPSO model are shown in Table 3. The error between the 146th iteration and the 147th iteration is larger than the minimum acceptable precision (0.00001), and the error between the 147th iteration and the 148th iteration is smaller than the minimum acceptable precision. The error of the iterative calculation results after 148 generations is also less than 0.00001. The results in Figure 3 and Table 3 indicate that QPSO found the optimal neural network weights and thresholds in the 147th generation.

The prediction results of different calculation models obtained from the Matlab software are shown in Figures 4 and 5. In Figures 4 and 5, the predicted error and the relative error value were obtained according to the predicted and actual values. The formula is as follows:

$$
\begin{aligned}
& E_{1}=\gamma_{p}-\gamma_{a}, \\
& E_{2}=\frac{\left|\gamma_{p}-\gamma_{a}\right|}{\gamma_{a}},
\end{aligned}
$$

where $E_{1}$ is the predicted error, $E_{2}$ is the relative error, $\gamma_{p}$ is the predicted value, and $\gamma_{a}$ is the actual cost.

The calculation error of the QPSO-BP model is very small, the maximum predicted error is only 2.79 , and the maximum relative error is only $1.39 \%$. This proved that QPSO-BP had high calculation accuracy, and this paper detailedly compares the calculation accuracy of different calculation models in Section 4.1.

The ratio of training set to test set might affect the prediction result. At present, the ratio of common training set and test set is $90 \%: 10 \%, 80 \%: 20 \%$, or $70 \%: 30 \%[39,40]$. Therefore, this paper calculated the influence of these three proportions on the prediction results, and the calculation results are shown in Table 4.

According to the calculation results in Table 4, it is not difficult to see that, with the decrease of the proportion of training sets, the calculation accuracy of the prediction results decreases continuously. However, when the proportion of training set is $70 \%$, the calculation accuracy of prediction results is $3.98 \%$. Compared with other methods (the multiple regression, $\mathrm{BP}, \mathrm{GA}-\mathrm{BP}$, and $\mathrm{PSO}-\mathrm{BP}$ ), the

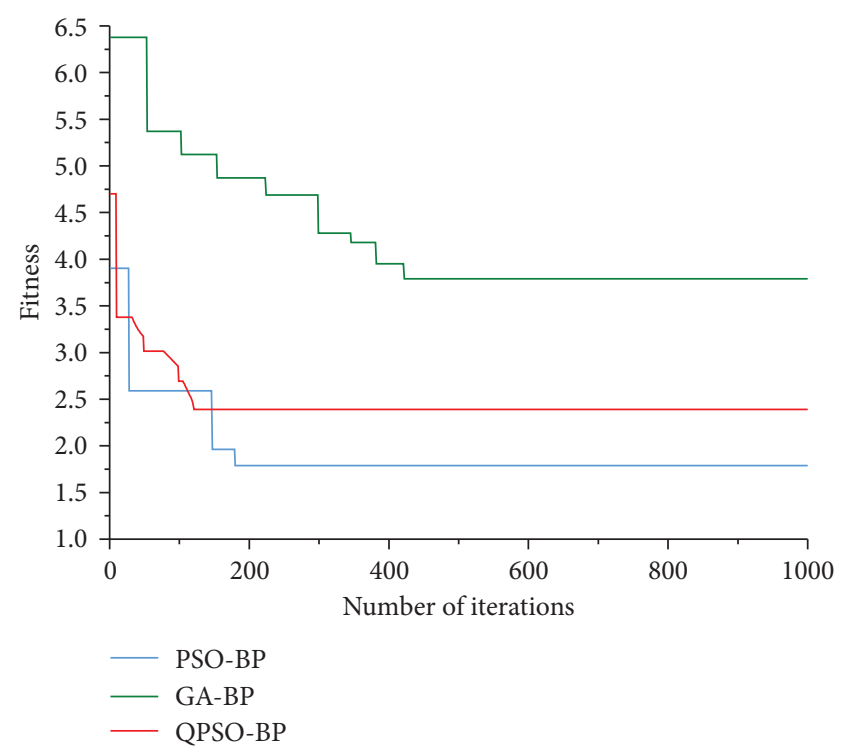

Figure 3: Convergence curves of the QPSO, GA and PSO models.

QPSO-BP with 70\% training set still has better calculation accuracy.

\section{Discussion}

In this paper, a rough set, the QPSO, and BP models were used to establish a prediction model of the construction cost of a subway shield project. This paper has the following limitations. (1) The primary indices of the index system in this study only refers to the engineering practices of the Chengdu Metro Line 11, Wuhan Metro Line 8, Tianjin Metro Line 7, and Wuhan Metro Line 21. If more projects are selected, the index system may change. (2) There is no complete analysis of the influence of input parameters on the prediction results in this paper. (3) Although the QPSO was successfully used to optimize the BP model, and its calculation accuracy was higher than that of common methods (the multiple regression, BP, PSO-BP, and GA-BP), many other optimization methods can also be used to optimize the BP model.

4.1. Error Analysis of the Different Computational Models. PSO and GA algorithms were used to optimize the BP model to determine the advantages of QPSO for optimizing the BP model. In the BP algorithm, the selected training function was traingda, the activation function of the hidden layer was logsig, and the activation function of the output layer was purelin [15-18]. We set the target error of training as $10^{-6}$ and the maximum number of iterations as 1000 . The learning rule of the network was the gradient descent method. In the GA algorithm, the number of individuals in the population was 50 , the maximum genetic algebra was 
TABLE 3: Optimization calculation process of the QPSO.

\begin{tabular}{lcccc}
\hline Iteration $(n)$ & Fitness $(n-1)$ & Fitness $(n)$ & Fitness $(n)-$ Fitness $(n-1)$ & Result \\
\hline 147 & 2.388799 & 2.388799 & $0<0.00001$ & Continue \\
148 & 2.388799 & 2.388807 & $0.000008<0.0001$ & Continue \\
149 & 2.388807 & 2.388807 & $0<0.0001$ & Continue \\
1000 & 2.388807 & 2.388807 & $0<0.0001$ & Stop \\
\hline
\end{tabular}

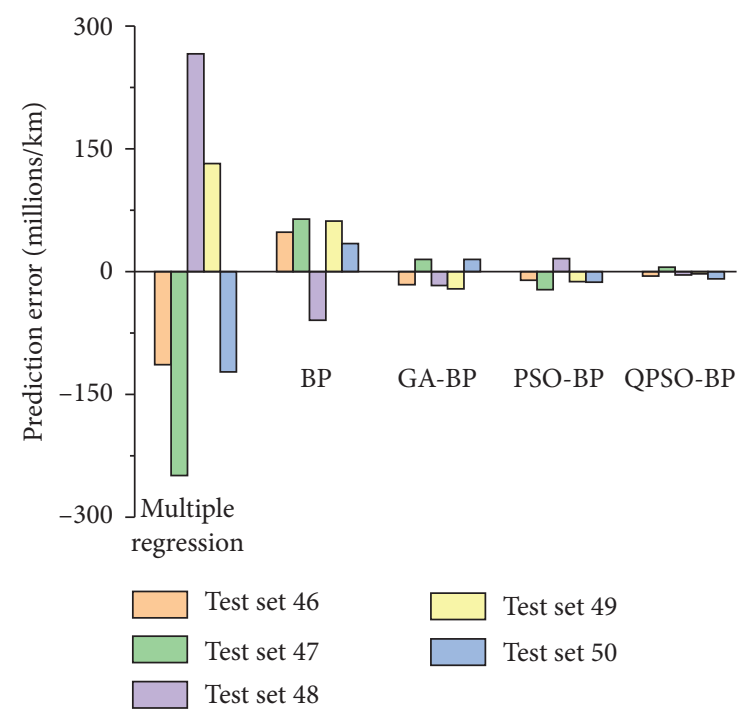

FIGURE 4: The predicted errors of different algorithms.

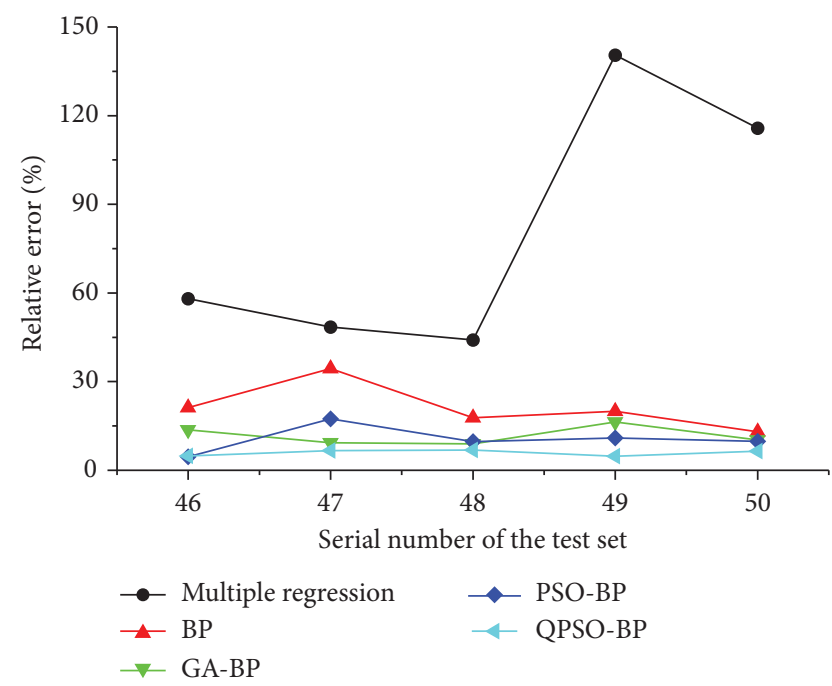

FIgURE 5: The relative errors of different algorithms.

TABle 4: Prediction results of different training and testing split ratio.

\begin{tabular}{lccc}
\hline $\begin{array}{l}\text { The training and testing split } \\
\text { ratio }\end{array}$ & $90 \%: 10 \%$ & $80 \%: 20 \%$ & $70 \%: 30 \%$ \\
\hline $\begin{array}{l}\text { Average absolute error } \\
\text { Average relative error (\%) }\end{array}$ & 5.278 & 7.313 & 12.763 \\
& 1.10 & 2.29 & 3.98 \\
\hline
\end{tabular}

1000 , the binary digits were 20 , and the generation gap was 0.9 [15]. In the PSO algorithm, the swarm size was 200, the personal learning coefficient and global learning coefficient were both 2 , the inertia weights decreased linearly from 0.9 to 0.4 , the minimum acceptance accuracy was 0.00001 , and the maximum number of iterations was 1000 [16]. In addition to population size and convergence conditions, the key parameter required for QPSO is only the scaling coefficient, whereas the GA algorithm and PSO require more parameters. Compared with the GA and PSO, the QPSO has the advantage of fewer parameters.

In addition, in the subsequent error calculation and analysis, we also used the $\mathrm{BP}$ algorithm and multivariate linear programming, a commonly used method in engineering practice, to predict the construction cost of subway shield engineering in this case study.

The convergence curves of the fitness function of the QPSO, GA, and PSO models are shown in Figure 3. The QPSO model converged after about 150 generations, the PSO model converged after about 270 generations, and the GA model converged after about 350 generations.

The results in Figure 3 and Table 3 indicate that QPSO found the optimal neural network weights and thresholds in the 147th generation. However, the GA and PSO only found the optimal neural network weights and thresholds in the 353rd and 264th generations, respectively. Thus, QPSO has a faster convergence speed than the GA and PSO.

According to Figures 4 and 5 in Section 3.2, the calculation errors of the multiple regression, BP, GA-BP, and PSO$\mathrm{BP}$ models are substantially larger than those of the QPSO-BP model. Therefore, the QPSO-BP model has the highest accuracy. In addition, the accuracy of the models follows the order QPSO-BP $>$ PSO-BP $>$ GA-BP $>$ BP $>$ multiple regression, which is similar to previous calculation results [22]. This phenomenon could prove the rationality of the case analysis results in this paper to a certain extent.

In order to further analyse the calculation accuracy of different calculation methods, we selected four common error analysis tools for machine learning, including the determination coefficient $\left(R^{2}\right)$, the mean absolute percentage error (MAPE), the root mean square error (RMSE), and the mean absolute error (MAE) to evaluate the accuracy of several methods. The results of the four types of errors are shown in Figure 6.

$R^{2}$ indicates the degree of correlation between the actual value and the predicted value; the calculation method is shown in equation (16). The closer $R^{2}$ is to 1 , the higher the correlation is and vice versa [41]:

$$
R^{2}=1-\frac{\sum_{i=1}^{N}\left(y_{i}^{\exp }-y_{i}^{\text {pre }}\right)^{2}}{\sum_{i=1}^{N}\left(y_{i}^{\exp }-y_{i}^{\overline{\exp }}\right)^{2}},
$$

where $y_{i}^{\exp }$ is the actual result, $y_{i}^{\text {pre }}$ is the predicted result, $y_{i}^{\overline{\exp }}$ is the average value of the actual results, and $N$ is the number of test sets. 


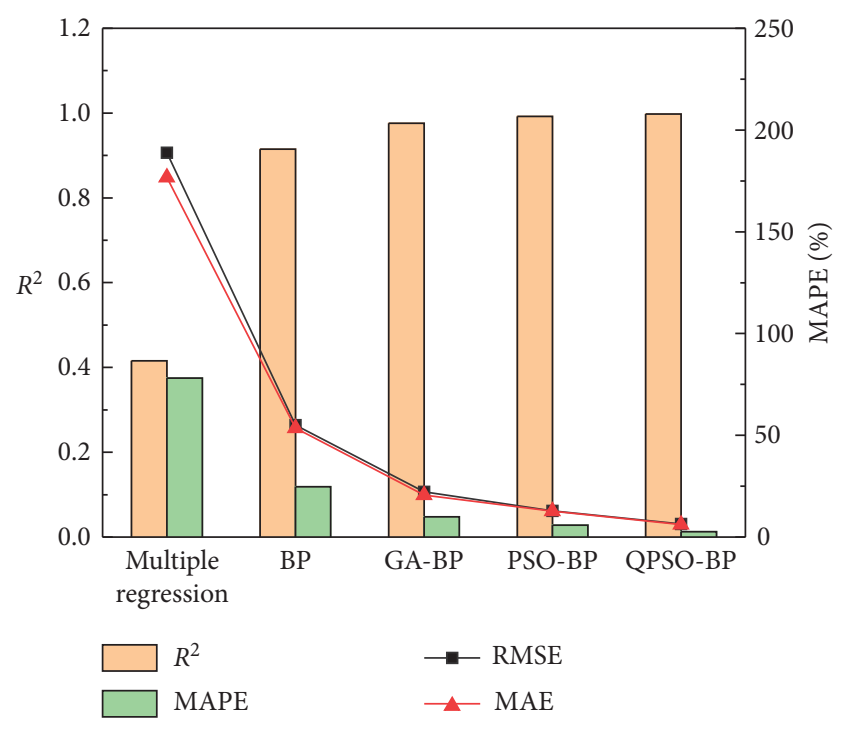

FiguRE 6: Error analysis results of the different calculation models.

As shown in Figure 6, the $R^{2}$ value of QPSO-BP is 0.9980, indicating that the predicted value obtained from the proposed model is very close to the actual value. Compared with the multiple regression analysis method, BP, GA-BP, and PSO-BP, the $R^{2}$ value of QPSO-BP is higher than those of multiple regression analysis, $\mathrm{BP}, \mathrm{GA}-\mathrm{BP}$, and $\mathrm{PSO}-\mathrm{BP}$, demonstrating that the proposed model has the best prediction results.

The MAPE represents the average relative error of the prediction results (equation (17)) [22, 41]. When the MAPE is $0 \%$, the prediction result is perfect. The higher the value of MAPE, the worse the accuracy of the prediction result:

$$
\text { MAPE }=\frac{1}{N} \sum_{i=1}^{N}\left|\frac{y_{i}^{\text {exp }}-y_{i}^{\text {pre }}}{y_{i}^{\exp }}\right| * 100 \% .
$$

The MAPE of QPSO-BP is the smallest (1.29\%), indicating that the prediction result of QPSO-BP is the best among all methods. The MAPE of multiple regression analysis, BP, GA-BP, and PSO-BP are $37.51 \%, 11.84 \%$, $4.75 \%$, and $2.83 \%$, respectively.

The RMSE measures the deviation between the predicted value and the actual value (equation (18)) [22]. The smaller the RMSE value, the smaller the deviation between the predicted value and the actual value and the higher the prediction accuracy:

$$
\text { RMSE }=\sqrt{\frac{1}{N} \sum_{i=1}^{N}\left(y_{i}^{\exp }-y_{i}^{\mathrm{pre}}\right)^{2}} .
$$

As shown in Figure 6, the RMSE value of QPSO-BP is 6.4363 , which is the lowest among all algorithms. The calculation results show that the proposed model provides the best prediction results.

The MAE is the average of the absolute values of the deviations between all predicted values and the arithmetic mean (equation (19)). The MAE avoids the problem of offset and describes the degree of data dispersion. The smaller the MAE value, the better the prediction accuracy:

$$
\operatorname{MAE}=\frac{1}{N} \sum_{i=1}^{N}\left|y_{i}^{\exp }-y_{i}^{\mathrm{pre}}\right| .
$$

The MAE value of QPSO-BP is the smallest among all models, indicating good dispersion of the prediction results and high prediction accuracy of this model. All five test sets achieved good prediction results.

We also conducted ten-fold cross-validation to test the accuracy of the algorithm [42] and verify whether the BP model was overfitted. The average accuracy of the ten-fold cross-validation is shown in Table 5.

The errors of ten calculation results are obviously smaller than the traditional BP and other three models, which shows the progressiveness of the calculation method. The accuracy is very high, and the average value of the maximum relative error calculated by the model proposed in this paper is $3.0009 \%$. In addition, the values of $R^{2}$, MAPE, RMSE, and MAE of ten calculation results are stable, which proves the stability of the proposed algorithm. Based on the above analysis, we deduced that the BP model of case analysis was not overfitted.

The number of input variables has a significant influence on the prediction results of the subway shield construction cost using an artificial intelligence method.

In Section 2.1.3 of this paper, the rough set method was used to concentrate the input variables, and eight factors (X2, X3, X4, X5, X7, X8, X13, and X14) which had great correlation with other input variables were deleted. In order to verify the rationality and effectiveness of using rough sets, we investigated the impact of deleting this indicator on the calculation results. The average $R^{2}$, MAPE, RMSE, and MAE of the ten prediction results changed slightly after these eight influencing factors were added, and the calculation time only increased by $54.7 \mathrm{~s}$. According to the basic principle of the rough set, we thought that these eight factors had great correlation with other factors. It was reasonable to delete these eight factors in Section 2.1.3.

4.2. Stability Analysis of Different Computational Models. Stability determines the reliability of the calculation model in prediction. In this paper, the standard deviation was used to judge the stability of the calculation model. Among the 45 training samples in Section 3.1, 30, 35, 40, and 45 samples were randomly selected as training sets, and the last 5 samples in Section 3.1 were used as test sets. The calculation was repeated 100 times, and the standard deviations of 5 test sets' prediction errors are shown in Table 6.

It can be seen from Table 6 that QPSO-BP has a low standard deviation, and the multiple regression had the largest standard deviation. With the increase of training sample size, the computational stability of QPSO-BP had been improved continuously. These results showed that QPSO-BP was more stable than other models. 
TABLE 5: Error results of the ten-fold cross-validation.

\begin{tabular}{lcccccccccc}
\hline No. & 1 & 2 & 3 & 4 & 5 & 6 & 7 & 8 & 9 & 10 \\
\hline$R^{2}$ & 0.9980 & 0.9981 & 0.9979 & 0.9975 & 0.9983 & 0.9980 & 0.9976 & 0.9976 & 0.9981 & 0.9982 \\
MAPE (\%) & 1.29 & 1.29 & 1.30 & 1.34 & 1.27 & 1.29 & 1.33 & 1.33 & 1.28 & 1.27 \\
RMSE & 6.4363 & 6.6789 & 6.5973 & 6.7910 & 6.4672 & 6.4780 & 6.3789 & 6.4789 & 6.2671 & 6.6781 \\
MAE & 6.078 & 6.3819 & 6.3891 & 6.4768 & 6.305 & 6.2673 & 6.3731 & 6.2781 & 5.7378 & 6.2789 \\
\hline
\end{tabular}

TABLE 6: Stability of calculation results under different sample numbers.

\begin{tabular}{lcccr}
\hline Model & 30 & 35 & 40 & 45 \\
\hline Multiple regression & 33.890 & 27.390 & 20.11 & 2.071 \\
BP & 1.890 & 1.631 & 0.451 & 0.938 \\
GA-BP & 0.817 & 0.639 & 0.432 & 0.381 \\
PSO-BP & 0.691 & 0.588 & 0.192 & 0.340 \\
QPSO-BP & 0.311 & 0.273 & 0.181 \\
\hline
\end{tabular}

TABLE 7: The topological structures of the different network models.

\begin{tabular}{|c|c|c|c|c|c|c|}
\hline Model & Number of hidden layers & Number of hidden layer nodes & $R^{2}$ & MAPE (\%) & RMSE & MAE \\
\hline \multirow{6}{*}{$\mathrm{BP}$} & \multirow{3}{*}{1} & 12 & 0.8531 & 37.89 & 263.8791 & 130.13 \\
\hline & & 15 & 0.8867 & 26.16 & 153.3810 & 78.31 \\
\hline & & 18 & 0.9152 & 11.84 & 54.9819 & 53.56 \\
\hline & \multirow{3}{*}{2} & $12-4$ & 0.8993 & 20.37 & 139.3117 & 58.48 \\
\hline & & $12-6$ & 0.9205 & 10.48 & 48.5281 & 34.76 \\
\hline & & $12-8$ & 0.9241 & 9.38 & 46.3189 & 32.18 \\
\hline \multirow{6}{*}{ GA-BP } & \multirow{3}{*}{1} & 12 & 0.9532 & 9.37 & 31.3819 & 28.37 \\
\hline & & 15 & 0.9648 & 6.39 & 27.9042 & 23.56 \\
\hline & & 18 & 0.9761 & 4.75 & 22.3047 & 20.68 \\
\hline & \multirow{3}{*}{2} & $12-4$ & 0.9713 & 5.17 & 27.3134 & 16.49 \\
\hline & & $12-6$ & 0.9848 & 4.31 & 20.8443 & 15.01 \\
\hline & & $12-8$ & 0.9910 & 3.48 & 17.3611 & 16.31 \\
\hline \multirow{6}{*}{ PSO-BP } & \multirow{3}{*}{1} & 12 & 0.9798 & 8.31 & 37.3428 & 26.44 \\
\hline & & 15 & 0.9831 & 4.48 & 23.189 & 15.31 \\
\hline & & 18 & 0.9920 & 2.83 & 12.8864 & 12.76 \\
\hline & \multirow{3}{*}{2} & $12-4$ & 0.9847 & 5.30 & 30.3197 & 17.50 \\
\hline & & $12-6$ & 0.9945 & 4.31 & 20.5610 & 12.89 \\
\hline & & $12-8$ & 0.9921 & 2.30 & 9.3819 & 6.15 \\
\hline \multirow{6}{*}{ QPSO-BP } & \multirow{3}{*}{1} & 12 & 0.9894 & 2.06 & 9.150 & 8.367 \\
\hline & & 15 & 0.9931 & 1.47 & 7.679 & 6.890 \\
\hline & & 18 & 0.9980 & 1.29 & 6.436 & 6.078 \\
\hline & \multirow{3}{*}{2} & $12-4$ & 0.9942 & 1.37 & 7.037 & 6.401 \\
\hline & & $12-6$ & 0.9982 & 1.25 & 5.936 & 5.568 \\
\hline & & $12-8$ & 0.9985 & 1.23 & 5.789 & 5.278 \\
\hline
\end{tabular}

4.3. Influence of the Topological Structure of the BP Model on the Calculation Results. To date, no unified and complete theoretical guidance has been developed for the selection of the BP neural network structure, and experience is commonly used for the selection. If the network structure is extensive, the training efficiency is not high, and there may be overfitting, resulting in low network performance and reduced fault tolerance. If the selection is too small, the network may not converge [43]. Therefore, we investigated the influence of the topological structure of different network models on the prediction results.

In the case study, the topological structure of the BP model was determined using equation (10). In line with the research results in related fields [44], we investigated the topological structure of seven different network models based on the QPSO-BP model; the results are shown in Table 7.

The average error of the calculation results of the QPSO-BP neural network model was very small, regardless of the topological structure of the network model. The results demonstrate the excellent performance of the QPSO-BP neural network model for forecasting the cost of construction projects. As observed in $[45,46]$, since the QPSO-BP neural network model has high precision for prediction and cross-validation results, the probability of overfitting or underfitting is reduced. When using the same model, the average error of the prediction results decreases with an increase in the number of hidden layers and nodes, but the calculation time also increases. 


\section{Conclusions}

Prior to construction, it is of great significance to perform an accurate and science-based prediction of the construction cost for the smooth implementation of a subway shield project. Due to the complexity of subway shield construction, many factors affect the project construction cost, and the cost prediction is complex and involves many nonlinear factors. Therefore, we decomposed the project using a project analysis method, obtained the factors influencing the cost of the subway shield project according to the Chinese national standards and expert opinion, and used rough set theory to reduce the number of influencing factors, thus avoiding redundancy between the engineering features. The case analysis of 50 subway shield projects in China indicated that the key factors affecting the project construction cost which included the type of shield machine, geological features, number of times that the shield machine entered and left the site, segment thickness, construction period, environmental characteristics, tunnel structure, project management ability of the owner, project management ability of the construction company, and financing structure of the project. Thus, several factors affected the project construction cost of the subway shield. The BP neural network optimized by QPSO was used to predict the construction cost of the subway shield. This method integrated the global convergence of QPSO and the ability to perform nonlinear analysis of the BP algorithm, thus improving the accuracy and stability of BP network training. The proposed model was well suited for the complex nonlinear problem of cost prediction in subway shield construction. The results of the absolute error, relative error, $R^{2}$, MAPE, RMSE, and MAE showed that the QPSO-BP model provided higher prediction accuracy than the multiple regression, BP, GA-BP, and PSO-BP methods.

In the future, scholars should develop an information database of the construction cost of subway shield projects to investigate the influencing factors more comprehensively. In addition, the use of other artificial intelligence algorithms to optimize the BP neural network is also worthy of further study.

\section{Data Availability}

The case analysis data used to support the findings of this study are available from the corresponding author upon request.

\section{Conflicts of Interest}

The authors declare that there are no conflicts of interest regarding the publication of this paper.

\section{Acknowledgments}

This study was supported by the Science and Technology Project of Wuhan Urban and Rural Construction Bureau, China (201943).

\section{References}

[1] J. Ahn, S. Cho, and D. H. Chung, "Development of a statistical analysis model to benchmark the energy use intensity of subway stations," Applied Energy, vol. 179, pp. 488-496, 2016.

[2] L. Hu, T. Li, and W. G. Qiu, "Study of dynamic prediction and control about tunnel construction schedule and cost," Journal of Railway Engineering Society, vol. 32, no. 1, pp. 115-121, 2015.

[3] M.-G. Li, J.-J. Chen, J.-H. Wang, and Y.-F. Zhu, "Comparative study of construction methods for deep excavations above shield tunnels," Tunnelling and Underground Space Technology, vol. 71, pp. 329-339, 2018.

[4] E. Bralic, "Experience in the use of FIDIC contracts on rail infrastructure projects," Gradevinar, vol. 71, no. 11, pp. 987-993, 2019.

[5] M. J. Wu and Q. L. Wu, "Present situation and prospect of mechanization construction of mountain highway tunnels," Journal of Chongqing Jiaotong University (Natural Science), vol. 39, no. 3, pp. 14-21, 2020.

[6] P. Wang, X. Kong, Z. Guo, and L. Hu, "Prediction of axis attitude deviation and deviation correction method based on data driven during shield tunneling," IEEE Access, vol. 7, pp. 163487-163501, 2019.

[7] S. Wang, Y. Mursalin, G. Lin et al., "Supply chain cost prediction for prefabricated building construction under uncertainty," Mathematical Problems in Engineering, vol. 2018, Article ID 4580651, , 2018.

[8] H. L. Chen, "Developing cost response models for companylevel cost flow forecasting of project-based corporations," Journal of Management in Engineering, vol. 23, no. 4, pp. 171-181, 2007.

[9] S. M. A. Tabei, M. Bagherpour, and A. Mahmoudi, “Application of fuzzy modelling to predict construction projects cash flow," Periodica Polytechnica Civil Engineering, vol. 63, no. 2, pp. 647-659, 2019.

[10] Y.-M. Cheng, C.-H. Yu, and H.-T. Wang, "Short-interval dynamic forecasting for actual $\mathrm{S}$-curve in the construction phase," Journal of Construction Engineering and Management, vol. 137, no. 11, pp. 933-941, 2011.

[11] S. Ding, H. Li, C. Su, J. Yu, and F. Jin, "Evolutionary artificial neural networks: a review," Artificial Intelligence Review, vol. 39, no. 3, pp. 251-260, 2013.

[12] R. Kumar, R. K. Aggarwal, and J. D. Sharma, "Energy analysis of a building using artificial neural network: a review," Energy and Buildings, vol. 65, pp. 352-358, 2013.

[13] O. Oyebode and D. Stretch, "Neural network modeling of hydrological systems: a review of implementation techniques," Natural Resource Modeling, vol. 32, no. 1, Article ID e12189, 2019.

[14] J. F. Mas and J. J. Flores, "The application of artificial neural networks to the analysis of remotely sensed data," International Journal of Remote Sensing, vol. 29, no. 3, pp. 617-663, 2008.

[15] L. Wen, J. Yu, and X. Zhao, "Electric power engineering cost predicting model based on the PCA-GA-BP," in Proceedings of the 3rd Annual International Workshop on Materials Science and Engineering, Guangzhou, China, September 2017.

[16] W. Liu and Z. Chi, "Research and application of PSO-based BP neural network in the project estimate of government investment," in Proceedings of the IITA International Conference on Control, Automation and Systems Engineering, Zhangjiajie, China, July 2009. 
[17] A. Khare and S. Rangnekar, "A review of particle swarm optimization and its applications in solar photovoltaic system," Applied Soft Computing, vol. 13, no. 5, pp. 2997-3006, 2013.

[18] W. Fang, J. Sun, Y. Ding, X. Wu, and W. Xu, "A review of quantum-behaved particle swarm optimization," IETE Technical Review, vol. 27, no. 4, pp. 336-348, 2010.

[19] D. Yumin and Z. Li, "Quantum behaved particle swarm optimization algorithm based on artificial fish swarm," Mathematical Problems in Engineering, vol. 2014, Article ID 592682, 2014.

[20] M. Xu, L. Zhang, B. Du, L. Zhang, Y. Fan, and D. Song, “A mutation operator accelerated quantum-behaved particle swarm optimization algorithm for hyperspectral endmember extraction," Remote Sensing, vol. 9, no. 3, p. 197, 2017.

[21] J. Sun, W. Fang, and W. Xu, "A quantum-behaved particle swarm optimization with diversity-guided mutation for the design of two-dimensional IIR digital filters," IEEE Transactions on Circuits and Systems II: Express Briefs, vol. 57, no. 2, pp. 141-145, 2010.

[22] Y. Du, M. Ding, and C. Zhou, "Phase angle-encoded and quantum-behaved particle swarm optimization applied to three-dimensional route planning for UAV," IEEE Transactions on Systems, Man, and Cybernetics-Part A: Systems and Humans, vol. 42, no. 2, pp. 511-526, 2011.

[23] H. Liu, H.-q. Tian, D.-f. Pan, and Y.-f. Li, "Forecasting models for wind speed using wavelet, wavelet packet, time series and Artificial Neural Networks," Applied Energy, vol. 107, pp. 191-208, 2013.

[24] Z. Pawlak and A. Skowron, "Rudiments of rough sets," Information Sciences, vol. 177, no. 1, pp. 3-27, 2007.

[25] T.-A. Shiau, M.-W. Huang, and W.-Y. Lin, "Developing an indicator system for measuring Taiwan's transport sustainability," International Journal of Sustainable Transportation, vol. 9, no. 2, pp. 81-92, 2015.

[26] X. Cheng, R. Long, H. Chen, and W. Li, "Green competitiveness evaluation of provinces in China based on correlation analysis and fuzzy rough set," Ecological Indicators, vol. 85, pp. 841-852, 2018.

[27] Y. Ge, F. Cao, Y. Du, V. C. Lakhan, Y. Wang, and D. Li, "Application of rough set-based analysis to extract spatial relationship indicator rules: an example of land use in Pearl River Delta," Journal of Geographical Sciences, vol. 21, no. 1, pp. 101-117, 2011.

[28] E. S. Andersen, "Value creation using the mission breakdown structure," International Journal of Project Management, vol. 32, no. 5, pp. 885-892, 2014.

[29] Y. Xue, X. Li, D. Qiu et al., "Stability evaluation for the excavation face of shield tunnel across the Yangtze River by multi-factor analysis," Geomechanics and Engineering, vol. 19, no. 3, pp. 283-293, 2019.

[30] Z. Y. Ilerisoy and M. E. Tuna, "Construction costs of tunnel form buildings," Gradevinar, vol. 65, no. 2, pp. 135-141, 2013.

[31] A. S. Soliño and J. M. Vassallo, "Using public-private partnerships to expand subways: Madrid-Barajas International Airport case study," Journal of Management in Engineering, vol. 25, no. 1, pp. 21-28, 2009.

[32] D. D. Fan, X. Y. Liu, X. C. Jing et al., "Causes analysis on wall leaking of metro foundation pit in Nantong water-rich sandy stratum," Tunnel Construction, vol. 41, pp. 225-231, 2020.

[33] F. Witlox and H. Tindemans, "The application of rough sets analysis in activity-based modelling, opportunities and constraints," Expert Systems with Applications, vol. 27, no. 4, pp. 585-592, 2004.
[34] K. A. Bakhla, V. Verma, M. Hembram et al., "Internal consistency and factor structure of 12-item general health questionnaire in visually impaired students," Industrial Psychiatry Journal, vol. 22, no. 2, p. 109, 2013.

[35] S. Gao, M. Zhou, Y. Wang, J. Cheng, H. Yachi, and J. Wang, "Dendritic neuron model with effective learning algorithms for classification, approximation, and prediction," IEEE Transactions on Neural Networks and Learning Systems, vol. 30, no. 2, pp. 601-614, 2019.

[36] W. Dong and M. Zhou, "A supervised learning and control method to improve particle swarm optimization algorithms," IEEE Transactions on Systems, Man, and Cybernetics: Systems, vol. 47, no. 7, pp. 1135-1148, 2017.

[37] J. Li, J. Zhang, C. Jiang, and M. Zhou, "Composite particle swarm optimizer with historical memory for function optimization," IEEE Transactions on Cybernetics, vol. 45, no. 10, pp. 2350-2363, 2015.

[38] F. Z. Qi, S. Q. Lin, and T. T. Yu, "A prediction model of stock price based on PCA and IFOA-BP neural network," Computer Applications and Software, vol. 37, no. 1, pp. 116-121+156, 2020.

[39] H. Peng, H. Wu, and J. W. Wang, "Research on the prediction of the water demand of construction engineering based on the BP neural network," Advances in Civil Engineering, vol. 2020, Article ID 8868817, , 2020.

[40] P. Zhang, R. P. Chen, and H. N. Wu, "Real-time analysis and regulation of EPB shield steering using random forest," Automation in Construction, vol. 106, Article ID 102860, 2019.

[41] M. A. Ahmadi, R. Haghbakhsh, R. Soleimani, and M. B. Bajestani, "Estimation of H2S solubility in ionic liquids using a rigorous method," The Journal of Supercritical Fluids, vol. 92, pp. 60-69, 2014.

[42] M. R. Pahlavan-Rad, K. Dahmardeh, M. Hadizadeh et al., "Prediction of soil water infiltration using multiple linear regression and random forest in a dry flood plain, eastern Iran," Catena, vol. 194, Article ID 104715, 2020.

[43] W. Liu, K. Wei, J. Xu, and X. Ji, "A hybrid algorithm for the reliability evaluation models of chemical systems," Quality and Reliability Engineering International, vol. 33, no. 7, pp. 1337-1349, 2017.

[44] R. Mo, G. L. Tian, and H. B. Sun, "Application of BP neural network with genetic algorithm to roughness prediction," Mechanical Science and Technology for Aerospace Engineering, vol. 34, no. 5, pp. 729-732, 2015.

[45] P. Barmpalexis, A. Karagianni, G. Karasavvaides et al., "Comparison of multi-linear regression, particle swarm optimization artificial neural networks and genetic programming in the development of mini-tablets," International Journal of Pharmaceutics, vol. 551, no. 1-2, pp. 166-176, 2018.

[46] Q. Meng, X. Ma, and Y. Zhou, "Forecasting of coal seam gas content by using support vector regression based on particle swarm optimization," Journal of Natural Gas Science and Engineering, vol. 21, pp. 71-78, 2014. 